\title{
La force d'obligation du langage (1938)
}

\section{Hans Lipps}

Traducteur : Simon Calenge

\section{OpenEdition}

\section{Journals}

Édition électronique

URL : http://journals.openedition.org/alter/657

DOI : 10.4000/alter.657

ISSN : 2558-7927

Éditeur :

Association ALTER, Archives Husserl (CNRS-UMR 8547)

Édition imprimée

Date de publication : 31 décembre 2018

Pagination : 215-229

ISBN : 978-2-9550449-4-0

ISSN : 1249-8947

\section{Référence électronique}

Hans Lipps, «La force d'obligation du langage (1938) », Alter [En ligne], 26 | 2018, mis en ligne le 31

décembre 2019, consulté le 26 mars 2020. URL : http://journals.openedition.org/alter/657 ; DOI :

https://doi.org/10.4000/alter.657 


\section{LA FORCE D'OBLIGATION ${ }^{1}$ DU LANGAGE (1938)}

Hans Lipps

[Hans Lipps est élève de Husserl, participant au premier cercle phénoménologique de Göttingen, dans lequel il discute avec Koyré, Reinach, Hering, et bien sûr Husserl lui-même. Cependant, Lipps ne suit pas l'orthodoxie husserlienne et s'oriente très tôt dans une compréhension toujours plus personnelle de la phénoménologie, qui

\footnotetext{
${ }^{1}$ [Afin de rendre la présentation plus claire, nous avons mis entre crochets les remarques du traducteur. Les notes sans crochet sont de l'auteur].

[Le terme allemand employé ici - « Verbindlichkeit » - déploie son sens dans une multitude de directions qu'il est difficile de toutes rapporter en français. «Verbindlichkeit » renvoie à « verbinden » : lier, joindre. La «Verbindlichkeit» renvoie ainsi au fait que l'usage de chaque mot nous lie non seulement à une idée, mais aussi à une communauté de locuteurs. Les paroles possèdent une «Verbindlichkeit » dans la mesure où se dépose en elles une multitude de décisions de discours, prises par les locuteurs présents et passés. Les mots ont ainsi une signification limitée, à laquelle est forcé de se plier tout locuteur. «Verbindlichkeit » signifie également une obligation morale : elle s'impose par exemple lorsqu'on est lié par une promesse ou par un engagement. Elle se présente alors comme cette puissance qui accompagne les agents et les pousse à tenir leurs engagements. Rapportée au langage, elle exprime la thèse de Lipps selon laquelle chaque parole est une promesse faite par le locuteur, mais dont la réalisation incombe à l'auditeur. Une même responsabilité est à l'œuvre dans ces deux sens de «Verbindlichkeit » : celle du locuteur qui, par la parole reçue des autres, répond aux choses, et celle de l'auditeur, qui doit à son tour répondre à celui qui lui parle. Comme en témoigne l'ensemble du texte, le fait que la langue oblige le locuteur à se plier à des décisions que le mot incarne, et le fait que chaque mot prononcé oblige l'auditeur à un accomplissement de sa signification, prennent l'aspect d'une force (Kraft) ou parfois d'une puissance (Potenz). La théorie de la signification de Lipps est ici, comme le remarque Fritjhof Rodi, une théorie énergétique. (Cf. Rodi, «Die energetische Bedeutungstheorie Hans Lipps », in Die Erfahrung der Bedeutsamkeit, Fribourg/Munich, Karl Alber Verlag, 2015, p. 130-144). Cette sémantique énergétique s'exprime dans le caractère d'obligation que porte chaque parole, obligeant, par une force propre, le locuteur et l'auditeur à se plier à une signification.

Pour traduire cette expression difficile, nous avons choisi l'expression "force d'obligation ». Le terme « obligation » renvoie à l'idée d'un devoir, lequel cependant ne vient pas de moi, d'une décision d'un sujet moral s' obligeant lui-même à partir d'une loi intérieure, mais trouve son origine dans un phénomène qui - devant moi «ob»-me lie « ligatio » à la nécessité d'accomplir quelque chose à partir de lui. L'expression «force d'obligation » permet ainsi de dire comment la parole qui vient d'en-face me lie au devoir d'accomplir quelque chose à partir d'elle. N.D.T.].
} 
le tourne vers l'herméneutique. Le cheminement de Lipps est alors parallèle à celui de Heidegger, qu'il lira avec attention, et dont il reprendra plusieurs traits dans le second tome de ses Recherches pour une phénoménologie de la connaissance. Ce premier livre de Lipps publié en 1927-1928 fut le commencement d'un itinéraire qui devait le mener dix ans plus tard à publier Recherches pour une logique herméneutique en 1938. Cet ouvrage témoigne de la conscience intime que Lipps avait de ce que doit être la pratique du discours. Il rend compte des rapports que doivent entretenir philosophie et linguistique, phénoménologie et herméneutique. L'article traduit ici date de la même année, et donne un aperçu condensé et rapide de ses thèses, en prenant toujours le langage comme thème privilégié. Le style de Lipps est reconnu difficile par son caractère fragmentaire. L'absence de système que revendique Lipps le conduit à un exposé qui s'apparente bien souvent à une liste de propriétés empiriquement constatées. C'est au lecteur qu'il revient chaque fois de découvrir la cohérence qui est cependant bien présente dans le texte] $]^{2}$.

On distingue les langues ${ }^{3}$ naturelles de la « langue » d'un calcul, en soulignant que l'appellation "langue » est seulement métaphorique dans le second cas. Le calcul est une opération qu'on doit mesurer à son concept - à la différence de la langue au sens d'une lingua naturelle, qui est ce qu'elle est dans une particularité absolue. Que la langue naturelle soit chaque fois une langue déterminée ne signifie pas la perte du général, mais seulement qu'en tant que langue, elle est toujours la langue de quelqu'un en particulier, en tant que langue générale par exemple, toujours celle d'un peuple. Les différences objectives que la linguistique comparative met en avant ne sont pas constitutives de la "diversité" des langues naturelles. Qu'il s'agisse de langues "naturelles" ne signifie pas qu'elles ne peuvent être employées que comme le moyen le plus proche pour accomplir quelque fonction qui pourrait être mieux remplie par ce qui aurait le principe de sa construction dans

\footnotetext{
${ }^{2}$ [Le texte présenté ici est repris de l'édition des œuvres complètes : H. Lipps, Werke 2, Francfortsur-le-Main, Klostermann, 1972, p. 107-120. Il fut publié pour la première fois en 1938 dans Blätter für deutsche Philosophie, Band 12, Heft 3, Berlin, Junker und Dünnhaupt Verlag, p. 282-292. Nous reproduisons la pagination des œuvres complètes. N.D.T.].

${ }^{3}$ [Le terme utilisé par Lipps est ici «Sprache », qui renvoie tout autant à la notion de langage qu'à la notion de langue déterminée. Dans la mesure où Lipps étudie ici le premier uniquement dans la mesure où il s'incarne dans la seconde, nous traduirons le plus souvent par « langue ». N.D.T.].
} 
une telle fonction, comme c'est le cas du calcul. Ce qu'il y a de "naturel" dans ces langues exprime plutôt quelque chose comme l'absence, par principe, de questionnement dans la manière dont elles sont parlées, perçues, et comprises. C'est le but concret qui distingue le calcul de la "langue" au sens originaire du terme : que l'homme ait le langage, que l'on se tienne par la langue que l'on parle dans une communauté avec d'autres.

Le calcul est un langage pour quelque chose. La communauté se constitue ici dans une compréhension qui est un pouvoir lire et déchiffrer : il s'agit d'un cercle d'initiés qui se comprennent sur des formules. On ne partage rien dans la langue du "calcul", qui n'est pas du tout parlée. Le calcul est une écriture de signes. Il signale [macht kenntlich]. On ne perçoit rien en lui ${ }^{4}$. La lecture de cette écriture se réfère à [108] une transposition objective. C'est en tant que mode de présentation que les calculs se distinguent. La formule, dans laquelle on inscrit ici des

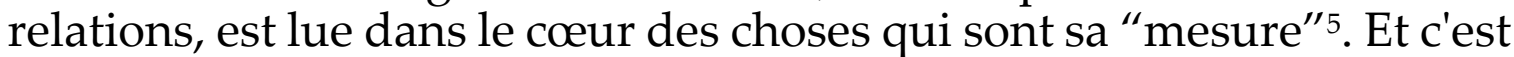
à l'aune de la chose même qu' on mesure la validité de la formule. Dans les signes du calcul, on symbolise d'après les choses ; les signes ne sont pas des "expressions" comme le mot qui se tient sous cette tension d'avoir à vouloir "dire" quelque chose ${ }^{6}$. Le calcul est lui-même une science qu'il s'agit d'apprendre, d'exercer, et pour le procédé de laquelle il importe d'acquérir la disposition interne de l'expert. Il accomplit quelque chose, à la différence du "pouvoir" [Können] du langage au sens strict qui se tient dans la clarté de son origine.

\footnotetext{
${ }^{4}$ En lien avec cette écriture de signes, Scholz déforme le mot prononcé en le réduisant à une combinaison de lettres. Dans cette conception on comprend que le mot tienne lieu de quelque chose à la manière d'un signe et soit à connaître dans cette visée. La signification du mot au sens strict tient cependant dans un rapport essentiel au fait d'être entendu et perçu. C'est justement la sonorité du mot qui renvoie à une origine de la langue que l'on ne peut perdre.

[Lipps parle ici de Heinrich Scholz, auteur d'un opuscule « Natürliche Sprache und Kunstsprache » publié dans le même volume des Blätter für deutsche Philosophie dans lequel Lipps a publié pour la première fois « Die Verbindlichkeit der Sprache ». Scholz défend la thèse selon laquelle l'exposé scientifique implique l'abandon de la langue naturelle au profit d'une langue artificielle. La première serait pleine de contradictions que Scholz se plait à décrire (cf. note 13 ci-dessous). La seconde serait suffisamment claire et précise pour permettre, sans contradiction, l'exposé de vérités scientifiques. Il semble que tout l'article de Lipps soit écrit contre la thèse de Scholz qui défend l'idée explicitement frégéenne d'une langue idéographique, que Scholz appelle langue du calcul : « Natürliche Sprache und Kunstsprache », Blätter für deutsche Philosophie, 1938, p. 70. N.D.T.].

${ }^{5}$ [L'usage des guillemets par Lipps est chaotique, employant tantôt des guillemets simples tantôt par des guillemets doubles. Nous suivons l'usage de Lipps, sauf lorsqu'il est question de citations, que nous mettons entre chevrons N.D.T.].

6 « La forme langagière interne des nombres est d'après leur origine et leur essence un affairement [Hantieren] » (Vossler, Geist und Kultur in der Sprache, 1925, p. 233). [Sur Vossler, voir notre note 11. N.D.T.].
} 
Le calcul est une présentation qui rend compte. Il signifie la mise en évidence critique de quelque chose à examiner. La chose [Sache] est déployée dans ses traits, examinée et mise en formule. Par un recentrement [Umzentrierung], en jugeant, on arrive à une présentation. La mise en mot d'une pensée a lieu par contre en tant que responsabilité. La pensée est alors accueillie. Cette responsabilité, telle qu'elle a lieu dans le langage au sens strict, est quelque chose d'autre que le simple fait de rendre compte.

Le mot signifie une responsabilité dans la mesure précisément où il est "expression". Répondre de quelque chose signifie que la chose est mise en mon pouvoir. Le mot ne tient pas seulement lieu pour quelque chose dans le but de le rendre connaissable - comme dans le symbole algébrique. À la différence de la définition d'un signe qui doit être déployée objectivement $e x$ definitione, et dont l'accomplissement requiert seulement le complément de mon intervention, c'est-à-dire [109] une praxis s'accomplissant à partir des choses, le mot est semantikos, dans la mesure où il éveille l'accomplissement de ce qu'il "signifie"7. Il porte quelqu'un à quelque chose, mais il ne donne pas quelque chose à quelqu'un. La signification du mot ne consiste pas seulement en la fixation de son appartenance à la parole, comme si elle lui était liée elle qui en est justement inséparable. La signification du mot consiste dans une force d'obligation [Verbindlichkeit] qui s'approprie le mot tel qu'il est généralement perçu. L'équivalence habituelle de la signification du mot au "concept" [Begriff] qui lui est lié a au moins ceci de juste que l'accueil du mot a lieu dans l'accomplissement de la prise [Griff] que le mot donne à connaître à quelqu'un. On correspond au mot en ce qu'on se lie [verbindet] à la prise indiquée par le mot, et qu'on reçoit quelque chose qu'il faut saisir. Et le mot se remplit [erfüllt sich], devient vrai, lorsque les choses se montrent à la lumière de la décision qu'il implique. Celle-ci détermine son interprétation. La signification verbale est cette relation produite par le langage, tracée dans le mot, frayant la voie à l'examen. La diversité réelle de ce qui est désigné en tant que «corps » [par exemple] montre également à quel point la

\footnotetext{
7 [La notion d'éveil est empruntée ici à Josef König - un élève de Georg Misch - qui s'efforce de concevoir un type d'expérience qui ne se réduit pas à la simple rencontre d'un sujet et d'un objet, mais au fait que quelque chose fasse effet sur moi et éveille en moi une impression qui, quoique subjective, permet l'attribution de propriétés spécifiques aux objets (Sein und Denken, Studien im Grenzgebiet von Logik, Ontologie und Sprachphilosophie, Halle, Max Niemeyer, 1937, p. 21). Lipps fait plus amplement référence à König dans Recherches pour une logique herméneutique, trad. S. Kristensen, Paris, Vrin, 2004, p. 92. N.D.T.].
} 
signification de tels termes ne peut être rendue présente qu'à partir du mot. Ce que le mot d'une langue signifie ne peut être "atteint" que par le mot d'une autre langue, dans les limites de cette dernière. Au mot, on ne peut rien attribuer de ce qui est objectif, c'est-à-dire de ce qui serait susceptible d'être montré sans le mot et qui lui serait seulement ensuite inféodé. Seul celui qui perçoit le mot accomplit la signification, non celui qui le prononce. On peut alors être l'autre pour soi-même, vouloir se dire soi-même quelque chose et chercher le mot qui dispose à accueillir les rapports permettant la saisie de quelque chose. Que le mot veuille être perçu se rapporte justement à l'accueil de sa signification. Lorsqu'on parle cependant d'une "compréhension" du mot dans sa signification, c'est à tort et en sous-main que l'on insère la compréhension objective de ce qui est médiatisé par le discours. [110] Dans cette compréhension, on est accaparé par l'autonomie [Selbstständigkeit] du mot, dans la mesure où l'on doit traduire ce qui est dit dans "sa" langue, et, en le méditant et en le pensant, se l'approprier.

Dans l'expression langagière, des relations ne sont pas saisies isolément pour être présentées comme dans la langue du calcul. Le mot signifie plutôt une accentuation qui condense et intensifie. Il est expression en tant que décision. La cohésion que la pensée reçoit une fois exprimée n'est pas une structure qui se trouve dans les choses et qui serait simplement mise en avant. La pensée en effet est simplement "accueillie" dans le mot, saisie et captée. Elle se noue dans le mot. Le sens est ce qui veut être perçu à travers le mot comme ce qui fixe pour la première fois le mouvement de sa signification. Ce que l'expression reçoit n'est pas une relation objective, mais ce par quoi on est mû, en tant que pensée, ce qui en tant que pensée ne peut être détaché de l'auto-accomplissement de l'existence. Le sens retentit à tel point que, dans ce qui est lu, la couche inférieure doit, pour être comprise, être entendue dans la manière dont elle s'exprime dans la courbe sonore de la phrase. Même dans un ordre imprimé par exemple, nous nous trouvons "abordés", quelque chose nous est "dit". Et la diction est choisie de sorte qu'un tel ordre corresponde par avance à une certaine situation [Lage], c'est-àdire 'accueille' les circonstances qui permettent de saisir le dispositif [Apparat] correspondant.

Le mot prononcé - dans la mesure où sa détermination vient d'une décision - se tient toujours en tension avec ce qui n'est pas relevé8. Ce

\footnotetext{
${ }^{8}$ Ce qui est ainsi perçu en demeurant inexprimé ne doit pas être confondu avec ce qui est compris à travers le mot, comme se trouvant entre les lignes et peut-être mal dissimulé par le mot. Ce qui est ainsi perçu n'est pas ce qu' on entend ici comme un sens "authentique" pouvant être objectivement indiqué, comme si la langue possédait un double fond. Ce sens "authentique" cependant n'est pas ici un sens plus profond, mais peut être un complément objectif laissé à la responsabilité de l'autre -
} 
qu'on ne relève pas peut trouver son expression dans le ton, et peut vibrer par des gestes sonores. Il est perceptible en cela d'une manière qui ne permet pas le malentendu, [111] mais qui ne peut pourtant être manifeste, et que le mot ne peut pas relever. L'obscurité ne se manifeste que dans l'ombre qui appartient, comme son revers, à la clarté de la force d'expression langagière.

Le mot prononcé est toujours perçu dans une nuance. Seul un accomplissement fugitif parvient à faire s'éveiller la perspective sous laquelle le mot veut être pris. Dans la mesure où le mot n'a pas de signification autonome, il ne peut pas non plus être question d'une 'diversité de sens' de "est" ou "chaque" par exemple9. Ce qui est chaque fois visé ne peut pas, en effet, être sujet au malentendu. 'L'indé-

dans la mesure où on compte sur une compréhension moyenne. (Compréhension et entente se rapportent toujours à un tel contenu visé.) - De même à l'inverse, comprendre un texte philosophique "littéralement" signifie qu'il doit être compris à partir du mot, c'est-à-dire à partir du sens qui n'est perceptible qu'en lui. On ne se rapporte pas alors à quelque champ objectif d'intérêt. L'emploi de termes philosophiques peut devenir trompeur pour l'approche courante des problèmes philosophiques. Dans une telle approche, on affiche les conceptions et les points de vue comme dans un slogan ; des "affaires" sont traitées. Aucun rapport originaire d'appel aux choses n'est cependant ici éveillé. La philosophie ne signifie pas une pensée qui évalue et raisonne sur les questions données dans le but d'une découverte de réponses et positions dans lesquelles on peut se tenir. La philosophie ne signifie pas l'acquisition d'une clarté sur quelque chose - comme s'il s'agissait ici de connaître les choses de manière critique dans leur juste signification. On ne recherche pas la possession de pensées philosophiques. Au contraire, on interroge ici ce qui ne correspond à aucun intérêt naturel. Rien ne peut être indiqué de ce qui pourrait donner le fondement de quelque "entente". C'est une attitude dans laquelle, en son absence de sol, on doit toujours à nouveau être transposé, poussé. [112] L'authenticité du logos philosophique - dans la mesure où il veut être un "vrai" discours - se manifeste dans ce qu'il y a d'obstiné, de massif dans la langue philosophique, dans la dureté circonstancielle de ses tournures. Le mot "ouvre" quelque chose à quelqu'un. Que quelque chose soit porté au langage est l'unique possibilité de le montrer à partir de soi-même. Seul le mot peut découvrir ce qui demeure caché à la saisie objective. L'originarité de la langue au sens strict fait que la compréhension de soi peut être développée de manière articulée et ininterrompue, qu'elle est à comprendre dans l'accomplissement du mot à partir de ses fondements. Une saisie [Fassung] immédiate s'oppose à une appréhension [Auffassung] objective. Le discours philosophique est un parler sans intervention de l'homme méditant et donc aussi sans menace d'équivoque comme celle de la langue détachée d'elle-même et visant au-delà de soi. Le va-et-vient dialectique est essentiel au philosopher - et pas seulement requis au sens où quelque chose vient au langage par plusieurs de ses aspects. Le pouvoir de la philosophie se détermine justement par la tension qui se produit dans le fait d'avoir à porter quelque chose à la parole. (J. König, « Das spezifische Können der Philosophie als eu legein », Blätter für deutsche Philosophie, X, 1936, p. 129 et suivantes).

${ }_{9}^{9}$ [Allusion à la critique par Heinrich Scholz de l'équivoque des mots de la langue, qui se trouve tout particulièrement dénoncée dans « Natürliche Sprache und Kunstsprache » sur deux exemples : « est » et « chaque ». Cette équivoque serait génératrice de malentendus, et à terme, de contradictions, ce qui rendrait finalement la langue naturelle impropre à l'usage de l'exposé scientifique. Lipps reconnaît l'existence d'une diversité d'emplois des mots, laquelle ne donne cependant pas lieu à malentendu ou contradiction : le mot possède une ouverture sur une multitude d'emplois diversifiés, entre lesquels il y aurait des liens de parentés, mais chaque emploi est déterminé en fonction de la situation de locution, et ne saurait donner lieu au malentendu que parce que la situation d'emploi est négligée. C'est cette situation d'emploi que Lipps tente ici de rappeler. N.D.T.]. 
termination' de la signification du mot ne signifie aucune imprécision ; c'est le caractère abyssal [Ungründige] de l'existence qui apparaît ici. Quand la compréhension du discours de l'autre s'en tient à la lettre, c'est alors qu'a lieu une déformation du mot. Chaque mot est plutôt compris à partir d'un milieu. Manquer le milieu engendre le malentendu, c'est-àdire la compréhension biaisée de ce qui est prononcé de manière visiblement marginale. Quand, à propos d'une couleur, on parle de "l'être encore vert", [112] il n'est ni possible ni nécessaire que soit expressément dit ce sur quoi le regard tombe, ce qu'il fait apparaître, ou ce en vue de quoi quelque chose est ici abordé. Le mot "encore" par exemple signifie quelque chose seulement dans le discours ; c'est seulement dans l'ajout réfléchi d'une détermination, par le mouvement qui va du locuteur à l'interlocuteur que quelque chose peut lui correspondre. "Certes" ne doit être compris qu'à partir des rapports qui appartiennent à la parole prononcée en général, dans la mesure où son principe se trouve dans autre chose, mais pas dans les choses ${ }^{10}$. La pensée est "saisie" dans le mot, se manifeste en ce qu'on peut en soi-même lui faire face - ce qui signifie "se dire quelque chose". La pensée n'est en effet pas seulement exprimée - comme si l'expression lui faisait face comme quelque chose qui lui serait indifférent - la pensée n'est libérée [entbunden] pour ellemême que dans l'expression.

\section{3}

[113] La forme interne du langage est visible dans ce en vue de quoi le sens est accueilli à travers le mot, c'est-à-dire dans ce qui subit une intensification dans l'expression. Ce qui distingue les langues, c'est ce qui est exprimé en général, ce par quoi on l'exprime, ce à l'intérieur de quoi et la manière dont l'autre est anticipé, ce qui doit être complété dans l'expression. L'orchestration de la langue (Vossler) ${ }^{11}$ varie. Le

\footnotetext{
${ }^{10}$ D'emblée ce n'est pas seulement le discours, mais aussi la pensée qui se soumet à une considération biaisée, lorsque - comme chez Scholz - elle est posée comme quelque chose d'absolu, dans la solitude d'un penser et dont il faut tirer les conséquences. Par où "certes", "mais non" ne pourrait exprimer quelque chose selon son mode de manifestation que pour le penser isolé. L'éclaircissement progressif de ce qui se pense a lieu cependant du fait que l'on peut devenir l'autre pour soi-même - dans l'échange des points de vue.

${ }^{11}$ [Karl Vossler est un philologue allemand spécialiste de romanistique. Par-delà ses études particulières sur Dante ou Racine, Vossler produisit une recherche théorique sur le devenir des langues, s'intéressant à la manière dont la littérature produit ses effets sur l'histoire de la langue. Cette influence de la littérature se produit tout particulièrement dans ce que Vossler appelle l'orchestration, ou l'instrumentation de la pensée par la langue. L'orchestration est le terme que choisit Vossler pour décrire le caractère national de chaque langue. Elle semble alors pouvoir désigner tout autant la mise en forme phonétique d'une pensée par une langue, que la répartition des
} 
caractère non systématique du langage s'exprime dans le fait qu'avec la traduction d'une langue dans une autre, un mot peut toujours plus ou moins "correspondre" à un autre, il y a transposition d'un "monde" dans un autre. Lors de cette transposition, on est guidé par le sens qui sonne à travers le mot, non par le regard fixé sur la nudité des choses. Dans la possibilité d'une telle traduction se manifeste également d'un autre côté l'universalité qui appartient à chaque langue au sens strict. La détermination et la décision qui leur appartiennent montrent à nouveau à quel point le monde de l'homme est chaque fois articulé différemment. On se rapporte à ce qui peut être dit en général dans une langue en s'appuyant sur le fait qu'on peut d'une certaine manière "tout" dire dans chaque langue. Ici, [dans ce qui peut être dit], on se correspond, et ce, dans le libre rapport dans lequel on peut se rencontrer, toucher la pensée prononcée par l'autre et, en elle-même, se tenir devant l'autre comme lui répondant. La "langue" du calcul, unifiée de manière chiffrée, transcrit l'essentiel en général. De manière routinière, quelque chose est "recouvert" par... (gedeckt) ${ }^{12}$. On ne doit pas comparer la traduction d'une langue dans une autre avec une telle reproduction, c'est-à-dire une correspondance démontrable par rapport à ce qui est objectif.

La force d'obligation [Verbindlichkeit] de la langue ne signifie pas quelque "validité", à la manière dont des relations par exemple sont valables, en l'occurrence pour un domaine dont les cas sont à mesurer à cette validité, à présenter et évaluer en elle. La validité générale se rapporte à l'application de quelque chose, par exemple d'un procédé [114] comme celui du calcul. On revendique la justesse, pour laquelle il y a des critères. Dans cette validité générale, il y a une insistance sur une absence spécifique d'obligation [Unverbindlichkeit]. On n'est pas soi-même appelé par ces opérations qui n'ont qu'à être exécutées. Alors que, dans ce que je ne dois faire que correctement, de manière appropriée, je suis délié de moi-même - comme c'est le cas pour les mathématiques qui ne sont qu'instrument, et pour l'activité de compter qui peut être accomplie de la manière la plus sûre par une machine, la

\footnotetext{
fonctions des mots dans les phrases et les interactions verbales, ou enfin, la répartition des postures des interlocuteurs au sein d'un dialogue. Cette orchestration semble ainsi correspondre à ce que Lipps décrit ici : le contenu exprimé, la forme phonique d'expression, l'horizon dans lequel on aborde l'autre et dans lequel il répond. Cf. Geist und Kultur in der Sprache, Heidelberg, Carl Winter's Universitätsbuchhandlung, 1925, p. 144. N.D.T.].

${ }^{12}$ [Lipps fait ici allusion à la compréhension husserlienne de la synthèse de recouvrement (Deckung) par laquelle une intention signitive coïncide avec l'intuition. Lipps décrit ici la manière dont l'expérience est recouverte par le langage qui en transpose l'essentiel dans ses intentions. Il critique ainsi une conception du langage comme image et traduction de l'expérience, qui recouvre l'expérience. N.D.T.].
} 
force d'obligation [Verbindlichkeit] de la pensée signifie en revanche qu'elle est toujours celle de quelqu'un et qu'elle le reste. Que des pensées puissent être étrangères à quelqu'un, que l'on puisse montrer quelque chose de soi-même en elles exprime la manière dont l'existence s'accomplit comme dans une démarche qui lui est propre. Les pensées veulent être "accueillies" ; elles signifient des décisions, dont nous avons à répondre. Leur "principe" se trouve en moi. La force d'obligation [Verbindlichkeit] de la langue se rapporte au déploiement qu'une pensée subit lorsque son articulation entre sous la pré-décision d'une langue déterminée ${ }^{13}$.

"Réponse" signifie en général qu'on a accueilli la parole de l'autre, que l'on s'est opposé à la parole de l'autre. La réponse est le mot provoqué par la parole d'un autre. Par conséquent, la responsabilité de la parole signifie [115] qu'elle est prononcée dans l'attente de la réponse de l'autre. Dans la réponse, c'est à cette attente que tout incombe et c'est à elle que l'on fait appel. Ici quelque chose a été "porté à la parole"14, est entré dans la lumière de la décision impliquée dans le mot. "Une pensée reçoit détermination et clarté uniquement par le rayonnement issu d'une force de pensée étrangère ». Humboldt avait à l'esprit la mise à l'épreuve d'une pensée, telle qu'elle a lieu dans le dialogue, où

\footnotetext{
${ }^{13}$ À partir de cette force d'obligation [Verbindlichkeit] du langage se résout l'objection de Scholz selon laquelle le langage serait contradictoire. On ne peut accuser la langue naturelle de contradictions qui n'apparaissent que lorsque, par exemple, le mot est réduit à une combinaison de lettres, comme lorsque sous une expression langagière telle que « ... peut être désigné par moins de $\mathrm{n}$ lettres » on réduit la langue naturelle à une signature de lettres. De même l'expression langagière "ne pas revenir à soi-même » n'est pas le fondement du paradoxe qui se laisse développer à partir des propriétés qui y sont nommées. - On pourrait cependant reprocher à la langue le fait qu'elle dissimule des contradictions. Mais comment devrait-elle les manifester ? Elle devrait le faire là où le mot donne des occasions pour la clarification d'un comprendre dont le fondement doit être trouvé ici dans le sens.

[Lipps pointe ici deux exemples que propose Heinrich Scholz pour défendre sa thèse selon laquelle la langue naturelle serait intrinsèquement contradictoire, et qui reprennent les descriptions des apories russelliennes touchant la théorie des ensembles. Dans l'article « Natürliche Sprache und Kunstsprache » mentionné ci-dessus (cf. note 4), Scholz développe deux arguments qu'il serait trop long d'exposer en détail ici. Le premier insiste sur les apories auxquelles mènent les langues naturelles lorsqu'il s'agit de concevoir les propriétés auto-imputables (la classe, qui contient les classes ne se contenant pas elles-mêmes, se contient-elle elle-même ?). Le second tente de montrer que la phrase « $x$ peut être désigné par moins de $n$ lettres » serait en réalité contradictoire. Scholz tente de prouver l'incapacité de la langue naturelle à être son propre métalangage. Il montre que les énoncés de grammaire « le nom propre désigne un individu » ne sont pas plus pourvus de sens que l'énoncé : « il y a un plus petit entier naturel qui ne peut être désigné par moins de 1000 lettres ». Cf. " Natürliche und Kunstsprache », op. cit., p. 64-65. N.D.T.].

${ }^{14}$ [L'expression choisie ici par Lipps «auf das Wort gebracht » est difficile à traduire. Il ne peut s'agir du simple fait de mettre quelque chose en mot (zum Wort gebracht), les guillemets insistant ici sur le caractère inhabituel de l'expression. "Aufs Wort», "Auf mein Wort», signifie "sur parole ». L'expression signifierait ici alors porter quelque chose à cette parole, et à la fois faire entrer quelque chose dans le champ d'une promesse à tenir. N.D.T.].
} 
la parole de l'un est accueillie et donnée en retour par l'objection de l'autre. Une force de pensée étrangère est cependant aussi consignée dans la langue que l'on parle, car c'est toujours une langue qui ne m'est que transmise. La puissance [Potenz] de la langue se trouve dans la manière dont les choses sont touchées par le mot et développées en étant contraintes à entrer dans le cercle de la signification du mot. La puissance [Potenz] du mot s'oppose à la simple fonction d'un signe. Le mot est quelque chose qui ne peut être accompli qu'à partir de son origine - et ce aussi sûrement qu'en même temps la tendance à la compréhension se déplace toujours plus [loin de cette origine], et que sa signification langagière s'est échappée pour devenir le plus souvent simple nom. Comme les choses, les signes veulent simplement être manipulés. Le mot est une réponse aux choses, dans la mesure où leur déploiement a lieu dans le medium du langage. Le mot retourne à l'abord des choses. Il est réponse parce qu'il accueille et redonne, en tant que prise et saisie. Il "touche", "veut dire" quelque chose, mais n'est pas simplement la désignation de quelque chose. Un rapport aux choses est affiché ici. Les choses sont ainsi placées dans la lumière d'une interprétation que le mot fait comprendre parce qu'il appartient à une langue déterminée.

Les paroles peuvent entrer dans un rapport de remplissement [Erfüllung $]^{15}$. Elles deviennent vraies dans le remplissement. Celui-ci n'est rien dont le mot pourrait faire l'expérience au sens où il se trouverait quelque chose qui correspond à [116] ce que le mot "veut dire" [meint], de sorte qu'il recevrait un recouvrement [Deckung] objectif et la preuve de sa validité. Prêter au remplissement une telle signification serait trompeur. (La conception du mot comme "expression", c'est-à-dire comme "signe" était déterminée par cette signification.) Avoir à se remplir, pour un mot, signifie cependant le contraire d'une telle justification recherchée dans les choses parce que le mot est justement ce qui est premier. Son remplissement signifie sa réalisation,

\footnotetext{
${ }^{15}$ [Lipps emploie à dessein le terme Erfüllung, en l'empruntant à Husserl. Mais son but est ici de mettre en valeur une équivoque créatrice de sens, qui a échappé à Husserl : remplir se dit, au sens phénoménologique de l'intention signitive vide que l'on remplit par l'intuition ; mais il est également possible de "remplir une tâche ou une fonction ». Compris en ce sens, le remplissement est réalisation d'une tâche et par extension, concernant la parole, réalisation d'une promesse. Nous avons conservé le sens de "remplissement » dans notre traduction pour garder le sens phénoménologique du remplissement que Lipps entend redéfinir. N.D.T.].
} 
parce que le mot est précisément une promesse [Verheissung]. Est "rempli" quelque chose dont l'ébauche [Vorzeichnung] est donnée dans le mot. La parole devient, ou est vraie, non à la suite de quelque correspondance avec les choses, mais en ce qu'elle manifeste sa force [Kraft] dans le remplissement. Ce à l'intérieur de quoi le mot se réalise se montre à la lumière de ce qu'il ébauche. L'intention [Intention] du mot qu'on nomme habituellement "viser" est, si on la regarde correctement, une force [Kraft] du mot. Elle se montre dans sa force d'obligation [Verbindlichkeit]. Dans la promesse par exemple, le remplissement du mot est donné à la puissance [Macht] de l'homme. Par sa parole, se porter garant de quelque chose devant quelqu'un, c'est-àdire se placer sous une parole donnée signifie en prendre la responsabilité. La parole est ici rendue vraie. Quelque chose est alors ramené aux rapports de la vie et dans les limites de sa forme typique : ce qui concerne la possibilité interne du mot en général. C'est dans cette perspective qu'il faut saisir le problème de ce qu'on appelle la signification du mot, parce que l'accomplissement de la signification d'un mot est son remplissement. On correspond à ce qui ici est perçu dans le mot, et ce, par l'accueil d'une perspective : le remplissement se manifeste comme un devenir-vrai du mot. Déjà dans l'être-entendu se manifeste une "puissance"16 [Macht] du mot prononcé, que le mot prétend imposer. [117] Le mot me lie [verbindet] à quelque chose ${ }^{17}$, et il n'en va pas autrement pour les mots comme "table", etc., que pour une sentence par exemple. Comprendre un mot comme "car" veut dire : se laisser transposer dans la disposition à recevoir une justification supplémentaire.

La conception du mot en tant que logos semantikos a complètement déterminé la manière scientifique de le concevoir. Cette conception se

\footnotetext{
${ }^{16}$ La ruse du slogan se fonde sur cette puissance. On y succombe. La force [Kraft] ouvrante du mot se retourne ici en une puissance dissimulatrice supplantant les choses. Le slogan s'assimile tout ce que chacun vise dans une direction et veut selon une modalité déterminée. Il mobilise une aspiration simplement prête, un vouloir pulsionnel engourdi, sans donner authentiquement quelque chose à connaître, [117] c'est-à-dire sans me laisser m'éveiller à quelque chose. Les affects qu'il libère se substituent à une responsabilité authentique accueillant librement le mot. Rien n'est authentiquement dit dans le slogan. Il fait simplement effet. On réagit aveuglément sans la participation supplémentaire que requiert pourtant l'accomplissement d'une signification. En tant qu'auditeur du slogan, on est objet d'une influence, mais on ne s'engage (verbindet) pas librement à partir d'une pensée. Le slogan aveugle sans faire entrer quelque chose dans la lumière. D'un autre côté, en tant que mot il fait appel à la liberté d'un accueil correspondant. On croit ici percevoir un appel - que l'on n'a justement pas besoin de suivre, puisqu'il semble confirmer à celui qui l'entend ce qu'il est déjà.

17 [Dans cette expression doit se lire l'équivoque que Lipps veut imposer : le mot me lie, c'est-àdire $m^{\prime}$ attache à quelque chose : il réalise un lien. Mais ce lien prend la forme pratique d'une tâche à réaliser : le mot $\mathrm{m}$ 'oblige à quelque chose. N.D.T.].
} 
trouve dans une orientation déterminée de la compréhension de soi de l'homme qui n'est pourtant pas essentielle au mot. C'est une conception d'emblée biaisée, que celle qui veut considérer les mots de la langue à partir de la connaissance des choses. Déjà le nom ${ }^{18}$ par exemple "est tourné" vers l'autre, il le "pose". C'est seulement à la suite de cette relation, c'est-à-dire une fois que quelqu'un est cité, que le nom a sa place dans le discours et peut être employé pour caractériser d'un homme. De manière générale, "nommer" signifie soumettre, c'est-àdire activer ce rapport de servitude (à la différence de la désignation qui se "rapporte" simplement à quelque chose, mais ne la touche pas et n'y accède pas véritablement comme le nom ; la désignation signifie un but qui se tient au service de la vie). Le rapport de l'homme au monde et à l'autre est mis en lumière dans la manière dont le mot est prononcé, entendu et compris. C'est justement ici que se manifeste la proximité intime du langage et de la nature de l'homme.

La malédiction appelle. "Appeler" - cela signifie : toucher quelque chose en le nommant. De cette manière, [118] ce que le mot circonscrit par un charme submerge quelqu'un. La prégnance du mot trouve ici son acmé. Et c'est dans la force [Kraft] même du mot que se trouve son devenir-vrai, son se-remplir. Le mot "est" déjà ce qu'il nomme, ce qui, en tant qu'étant partout et nulle part, peut aussi, pour cette raison, être prélevé en le citant; ce à quoi on peut s'enjoindre soi-même ou à quoi on peut enjoindre un autre; ce par quoi on peut être ravi dans la mesure où cela submerge. Le mot est déjà ce qu'il nomme de la même manière que quelque chose se présente - est "produit" - dans les pratiques de la magie, dans les traits des masques. Ce n'est pas comme si la simple image, c'est-à-dire le simple nom, pouvait être confondu avec la chose. Mais ce qui est compris par nous comme sa réalité vaut ici comme la manifestation d'une "essence", dont on ne doit pas venir à bout «en comprenant ». Les choses ne se montrent pas à quelqu'un sous des aspects, c'est-à-dire dans un quoi qui se fonderait sur les cohésions naturelles. On ne dispose pas librement des choses dans leur mise en concept. On est sans véritable "position", toujours asservi aux choses. Si on ne fait que les toucher, les nommer, on les manque, passant à côté de ce qui est nommé.

On ne doit pas séparer le langage d'une manière particulière de l'être affecté [durchgestimmtsein] par le monde. Un mode d'être fondamental [comme celui de cette affection] se manifeste dans le fait

18 [Lipps parle ici visiblement du nom propre au sens usuel par lequel on désigne une personne. N.D.T.]. 
qu'aucun n'est plus "juste"19 que l'autre, et dans le fait qu'aucun ne peut être aboli dans un autre. On ne confère pas au mot une force [Kraft] inconcevable et occulte, comme si ici, en regardant correctement, les limites du mot et ce qu'il accomplit véritablement étaient méconnus. C'est seulement en tirant vers soi ces usages apparemment marginaux du mot, comme la malédiction, le proverbe, etc., que la signification du mot au sens que nous lui donnons se laisse comprendre seulement comme une modification et une organisation de cette même structure fondamentale. La puissance [Macht] ouvrante du mot, la force [Kraft] conceptuelle de la langue est de même origine que cette vis magica.

Ainsi l'impression [Eindruck], par exemple, est quelque chose qui est appelé par ce que dit le mot. Un mot comme "lisse" "sonne comme si on le sentait $»^{20}$. Ici, il n'est nullement question, pour Herder, de toucher une ressemblance extérieure et en même temps biaisée. [119]. "Comme si" ne veut pas dire ici que quelque chose devrait être indiqué de manière allégorique, comme si dans la conscience de l'imprécision d'une telle tentative il était question de traduire dans un autre domaine de réalité. Cela se rapporte plutôt à la force [Kraft] expressive du mot. La question est de savoir si cette force [Kraft] est seulement traduite ou si elle est précisément touchée dans ce "comme si".

C'est avant tout seulement l'impression que fait sur moi ce qu'il y a de plus divers qui peut aussi être exprimée - et non ce qu'on a comme sensation dans les doigts, non ce qu'on reconnaît dans la sensation, ni ce en quoi on le reconnaît. [Ce qui est exprimé n'est pas] à identifier objectivement en direction de ce qui s'atteste dans la sensation. Qu'une impression en rappelle une autre veut dire autre chose que l'avoir de manière mnésique dans les doigts, comme la manière dont la craie est sentie. Que quelque chose fasse ainsi effet sur moi, par exemple "soit" comme... ne signifie pas une recognition identifiante ${ }^{21}$. Le souvenir n'est pas une faculté pratique. Une "ressemblance" se trouve dans le

\footnotetext{
19 [La relation de « justesse » désignée ici par Lipps, décrit une simple correspondance entre le mot et son objet. (Cf. Lipps, Untersuchungen zur Phänomenologie der Erkenntnis, Francfort-sur-le-Main, Klostermann, 1976, p. 43-44). Il s'agit de la capacité du mot à toucher l'objet visé. N.D.T.].

${ }^{20}$ [Lipps cite ici Herder, Traité sur l'origine des langues, trad. L. Duvoy, Paris, Allia, 2010, p. 88 : « Les mots parfum, son, sucré, amer, âcre, etc., sonnent tous comme si on les sentait ». Le terme employé par Herder, et repris par Lipps ici est fühlen. Celui-ci renvoie moins à la sensation [Empfindung] qu'au sentiment, ou à l'impression. N.D.T.].

${ }^{21}$ [Lipps fait encore une fois allusion ici à la pensée de Josef König, qui, dans Sein und Denken définit l'impression comme un faire-tel-effet. Par l'impression, les choses les plus diverses font effet sur moi. Cet effet déterminé de l'impression n'est pas alors une détermination subjective de mon esprit, mais une détermination de l'être des choses. En l'occurrence, il s'agit de la détermination adverbiale de l'être : une pièce - même remplie - peut donner une impression de vide. Le vide est la manière adverbiale d'être de la pièce, elle est comme vide. N.D.T.].
} 
fait qu'avec l'un, l'autre vient à l'esprit. C'est seulement dans cet êtresouvenu que s'éclaire l'impression. Alors qu'on suit objectivement la sensation [Empfindung], que l'on suit en elle le renvoi à quelque chose, alors qu'ici "faire l'expérience de quelque chose" signifie justement suivre la piste, alors que, suivant les voies, on s'engage dans la réalité s'annonçant dans la sensation, une impression [Eindruck] requiert justement d'être élevée dans sa puretéé2 ${ }^{2}$. 'est seulement en s'éloignant de la concrétion de ce qui fait effet d'une manière déterminée que se découvre le noyau valide de l'impression. On doit ici devenir libre pour le souvenir au sens d'une anamnèse, par laquelle l'un entre sous la lumière de l'autre. L'élucidation d'une impression a lieu cependant à partir de moi, dans la mesure où je m'éveille à partir de moi-même à quelque chose. Faire effet sur moi signifie : être abordé par quelque chose. Le souvenir est alors provoqué. Ce qui s'élève ainsi dans le devenir éveillé du souvenir dans sa pureté ne subit de fixation que dans le mot, [120] dans la mesure justement où l'impression «est comme scellée par le son et prise en possession » (Nietzsche). Le mot "sonne" comme si on le sentait, parce qu'il est perçu en tant que mot, ce qui veut dire que le mot n'est pas simplement "entendu" - comme la structure matérielle des choses qui peut se manifester de manière audible. À la différence de l'audition fuyante du son que quelqu'un peut avoir à l'oreille, la perception signifie un accueil, c'est-à-dire un laisser sonner en soi-même, de sorte que de son côté, on peut y venir et y revenir. On perçoit le mot "dans l'intention de sa signifiance", c'està-dire en tant que quelque chose qui m'est dit. En ce que je perçois le mot dans son articulation sonore, chaque possibilité d'un effet sur moi est indiquée dans le sens d'une impression. En tant qu'expression, le mot "est" ici ce qui est exprimé, mis en liberté, saisi par l'appel. Ce qui, dans le mot, oblige, [das Verbindliche] se manifeste en ceci, que ce qui est saisi se trouve de quelque manière dans le mot. Mais le mot sonne comme si on le sentait : car ce qui est éveillé en moi est le souvenir dans la direction duquel une impression se condense en s'intensifiant. C'est justement ceci qui permet son partage : que la détermination condensant l'impression ait lieu comme sa spiritualisation [Vergeistigung].

\footnotetext{
22 [Hans Lipps distingue la sensation [Empfindung] de l'impression [Eindruck], la première établissant une relation objective en direction de la chose, la seconde ouvrant une détermination adverbiale de la chose. Si la sensation me rapporte à la chose et à son quid, l'impression me rapporte à la chose dans son quomodo. Il faut noter ici que, pour Lipps, il ne saurait jamais y avoir de sensation sans impression - et réciproquement. Cf. H. Lipps, Die menschliche Natur, Francfort-sur-leMain, Klostermann, 1976, p. 76 et p. 89, ainsi que Recherches pour une logique herméneutique, op. cit., p. 90-100. N.D.T.].
} 
Hans Lipps, « La force d'obligation du langage »

Si la parole articulée ne peut être simplement ramenée à un geste sonore, celui-ci est malgré tout sur la voie qui mène au langage. Le geste intensifie et condense, lui aussi, ce qui était d'abord indéterminé et n'était qu'une impression floue, incertaine d'elle-même. De même qu'il faut remarquer, à propos des traits transversaux dans la langue et des gestes, qu'il y a peu de gestes - dans l'organisation diverse de leur exécution - dans lesquels nos affects sonnent, de même il n'y a que peu de racines linguistiques qui, en tant que centres ordonnateurs, conçoivent le monde dans la diversité de ses phénomènes; de même encore l'homogénéité de l'homme est le principe des souvenirs qui sont appelés par la captation de l'impression dans le mot.

Traduit de l'allemand par Simon Calenge 